\title{
Record Ultra-low Phase Noise 12 GHz Signal Generation with a Fiber Optical Frequency Comb and Measurement
}

\author{
Xiaopeng Xie ${ }^{1}$, Romain Bouchand ${ }^{1}$, Daniele Nicolodi ${ }^{1}$, Michele Giunta ${ }^{2}$, Wolfgang Hänsel ${ }^{2}$, Matthias Lezius ${ }^{2}$, \\ Ronald Holzwarth ${ }^{2}$, Abhay Joshi ${ }^{3}$, Shubhashish Datta ${ }^{3}$, Christophe Alexandre ${ }^{4}$, Pierre-Alain Tremblin ${ }^{5}$, \\ Giorgio Santarelli ${ }^{1,5}$, and Yann Le Coq ${ }^{1 *}$ \\ ${ }^{1}$ LNE-SYRTE, Observatoire de Paris, CNRS, UPMC, 61 avenue de I'Observatoire, 75014 Paris, France. \\ ${ }^{2}$ Menlo Systems GmbH, Am Klopferspitz 19a, D-82152 Martinsried, Germany \\ ${ }^{3}$ Discovery Semiconductors Inc., 119 Silvia street, Ewing, New Jersery 08628, USA \\ ${ }^{4}$ LNE-CNAM, CEDRIC Laboratory, 1 rue Gaston Boissier, 75015 Paris, France \\ ${ }^{5}$ Laboratoire Photonique, Numérique et Nanosciences, IOGS - CNRS - Université de Bordeauxl, Rue F. Mitterand,33400 Talence, France \\ *E-mail address: yann.lecoq@obspm.fr
}

\begin{abstract}
We demonstrate a $12 \mathrm{GHz}$ signal with a record absolute phase noise of $-167 \mathrm{dBc} / \mathrm{Hz}$ at $10 \mathrm{kHz}$ and $-170 \mathrm{dBc} / \mathrm{Hz}$ at $100 \mathrm{kHz}$. We have developed a specific measurement setup for charactering this ultra-low phase noise.

OCIS codes: (140.4050) Mode-locked laser; (230.5160) Photodetectors; (350.4010) Microwave; (120.5050) Phase measurement.
\end{abstract}

\section{Introduction}

Microwave signals with ultra-low phase noise are of great interested in the fields of radar, telecommunication, deepspace navigation, very long baseline interferometry and precision metrology [1]. Especially, the microwave signals extracted from the optical frequency comb possess extremely low phase noise not only at the close-to-carrier Fourier frequencies but also at the far-from-carrier Fourier frequencies [2-6].

In this contribution, we will report on our use of a fiber optical frequency comb locked to a CW ultra-stable laser to generate a $12 \mathrm{GHz}$ carrier microwave signal with record low absolute phase noise levels of $-167 \mathrm{dBc} / \mathrm{Hz}$ at 10 $\mathrm{kHz}$ and $-170 \mathrm{dBc} / \mathrm{Hz}$ at $100 \mathrm{kHz}$ Fourier frequencies. This absolute phase noise is $10 \mathrm{~dB}$ lower than all the previously reported data using a similar approach [2-6]. Furthermore, this phase noise level is also comparable or even lower than that of signals generated by the best sapphire whispering-gallery microwave oscillator. Measuring such ultra-low phase noise is in itself an interesting challenge. We have setup for this purpose a heterodyne crosscorrelation technique with ultra-low measurement noise that uses two accessory statistically independent microwave sources as references.

\section{Principle and experimental setup}

Fig.1 shows the ultra-low phase noise $12 \mathrm{GHz}$ signal generation setup. A fast seft-starting Er-doped fiber-based optical frequency comb with $250 \mathrm{MHz}$ repetition rate is phase locked to an ultra-stable $1542 \mathrm{~nm}$ CW laser reference. A specially designed highly linear photodiode is used to convert the locked optical pulses train to electrical signals, generating harmonics of the comb repetition rate. The frequency comb thus transfers the spectral purity of the stateof-art ultra-stable CW laser to the microwave domain.

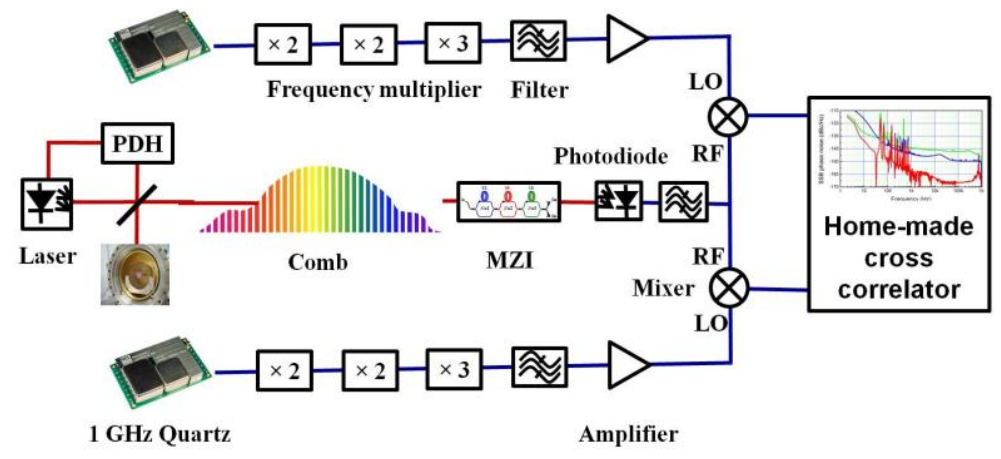

Fig. 1: Schematic of the ultra-low phase noise $12 \mathrm{GHs}$ signal generation system and phase noise measurement set-up.

Several key technologies are applied to get the record ultra-low phase noise result. An external repetition rate multiplier redistributes the photocurrent to the harmonics at $12 \mathrm{GHz}$, greatly increasing the signal to noise limit [7]. Furthermore, as the shot noise limit depends on the optical pulse duration [8], a piece of dispersion compensated 
fiber is introduced to compress optical pulse on the photodiode to less than 1ps. As the comb's amplitude noise produces excess phase noise through amplitude phase conversion in the photodetector, we need to decrease this effect by 1) decreasing the combs' amplitude noise with passive optimization combined with an active servo loop 2) operate the photodetector at a "magic point" where non-linear saturation effect exactly cancel the amplitude-tophase conversion noise for the $12 \mathrm{GHz}$ signal $[9,10]$. Rejection of amplitude noise by more than $30 \mathrm{~dB}$ and up to 50 are typically obtained and used.

To measure the resulting ultra-low microwave phase noise, we use a heterodyne cross-correlation technique, where two extra statistically independent microwave sources are introduced to implement a three cornered hat comparison (Fig.1). These two extra $12 \mathrm{GHz}$ reference sources are obtained by multiplication of commercial $1 \mathrm{GHz}$ oscillators. The two beat-note signals (near $10 \mathrm{MHz}$ ) obtained by beating these sources against the comb-generated signal are sent to a FPGA-based home-made system. They are sampled by fast analog-to-digital converters, digitally down-converted and computer processed to generate two time dependant phase comparison data sets. Crosscorrelation of the these two phase noise data sets reveals the power spectral density of the phase noise of the $12 \mathrm{GHz}$ signal that we want to characterize. The uncorrelated noise from the two accessory reference sources is rejected and contributes only to the uncertainty of the estimates of the phase noise power spectra density of the signal under test. This uncertainly averages down with the square root of the inverse of the measurement time.

\section{Results and discussion}

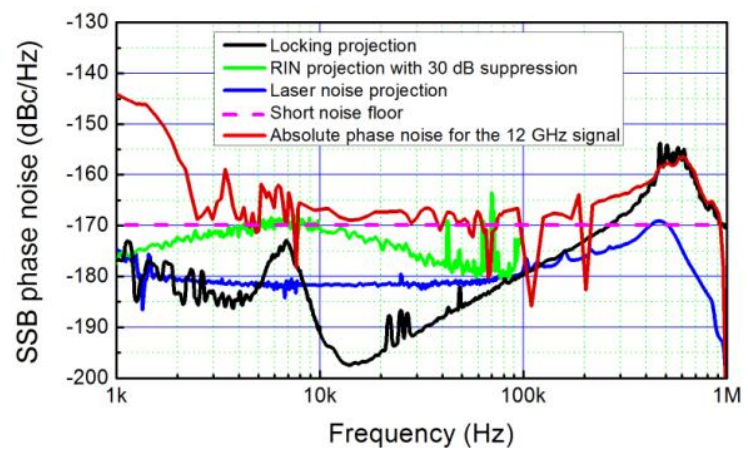

Fig. 2: Absolute SSB phase noise for the $12 \mathrm{GHz}$ signal extracted from the fiber frequency comb and limitations analysis.

Fig.2 shows the absolute phase noise power spectral density measured by the home-made cross-correlator after several hours' average. The red curve represents the absolute phase noise of the $12 \mathrm{GHz}$ signal generated with the fiber optical frequency comb. Other data sets, using frequency comb-based systems as auxiliary sources also show phase noise at $1 \mathrm{~Hz}$ Fourier frequency below $-100 \mathrm{dBc} / \mathrm{Hz}$. Blue, black and green traces show some identified contribution to this phase noise. The absolute phase noise at the $100 \mathrm{kHz}$ Fourier frequency is limited by the shot noise floor, which we plan to improve in the near future by using higher power fs-laser combined with higher power handling photodiodes. Ultra-low phase noise $-180 \mathrm{dBc} / \mathrm{Hz}$ at $100 \mathrm{kHz}$ seams within reach for the next generation system currently under development.

The authors would like to thanks Dawei Li, Jose Pinto and Michel Lours for the frequency chain implementation. This work is funded by the DARPA PULSE program (PureComb project), The FIRST-TF Labex and the Eurostar Eureka program (STAMIDOF project).

\section{References}

[1] J. Millo, M. Abgrall, M. Lours, E. M. L. English, H. Jiang, J. Guéna, A. Clairon, S. Bize, Y. Le Coq, and G. Santarelli, Appl. Phys. Lett. 94, 141105 (2009).

[2] A. Bartels, S. A. Diddams, C. W. Oates, G. Wilpers, J. C. Bergquist, W. H. Oskay, and L. Hollberg, Opt. Lett. 30, 667-669 (2005).

[3] J. Millo, R. Boudot, M. Lours, P. Y. Bourgeois, A. N. Luiten, Y. Le Coq, Y. Kersalé, and G. Santarelli, Opt. Lett. 34, 3707-3709 (2009).

[4] T. M. Fortier, M. S. Kirchner, F. Quinlan, J. Taylor, J. C. Bergquist, T. Rosenband, N. Lemke, A. Ludlow, Y. Jiang, C. W. Oates, and S. A. Diddams, Nat. Photonics 5, 425-429 (2011).

[5] A. Didier, J. Millo, S. Grop, B. Dubois, E. Bigler, E. Rubiola, C. Lacroûte, and Y. Kersalé, Appl. Opt. 54, $3682-3686$ (2015)

[6] E. P. Campa, G. Buchs, S. Kundermann, L. Balet, and S. Lecomte, Opt. Express 23, 32441-32451 (2015)

[7] A. Haboucha, W. Zhang, T. Li, M. Lours, A. N. Luiten, Y. Le Coq, and G. Santarelli, Opt. Lett. 36, 3654-3656 (2011).

[8] F. Quinlan, T. M. Fortier, H. Jiang, A. Hati, C. Nelson, Y. Fu, J. C. Campbell, and S. A. Diddams, Nat. Photonics 7, 290-293 (2013).

[9] W. Zhang, T. Li, M. Lours, S. Seidelin, G. Santarelli, and Y. Le Coq, Appl. Phys. B 106, 301-308 (2012).

[10] W. Zhang, S. Seidelin, A. Joshi, S. Datta, G. Santarelli, and Y. Le Coq, Opt. Lett. 39, 1204-1207 (2014) 